\title{
COMMENTARY
}

\section{Witnessing the last supper of uncultivated microbial cells with Raman-FISH}

\author{
Josh D Neufeld and J Colin Murrell
}

The ISME Journal (2007) 1, 269-270; doi:10.1038/ ismej.2007.55; published online 28 June 2007

Hunched over his microscope, Antonie van Leeuwenhoek discovered bacteria over 300 years ago, commenting that 'there were many very little living animalcules, very prettily a-moving'. Microbiology has come a long way since then. Modern environmental microbiologists continue to experience the thrill of discovery by studying the function of uncultivated microorganisms with increasingly creative methodologies (Neufeld et al., 2007). One of the goals is to link metabolic processes in the environment with particular microbial species, thus reducing the 'terra incognito' of microbial diversity and its implications.

Ironically, it is by means of a microscope that some of the most innovative approaches for linking diversity and function are still being realized today. One such breakthrough comes from Huang et al. (2007), who have added an elegant new tool to the ever-expanding toolbox available to microbial ecologists. These authors combined stable-isotope probing (SIP; Radajewski et al., 2000), Raman microscopy (for example, Huang et al., 2004) and fluorescent in situ hybridization (FISH; reviewed in Wagner et al., 2003) to establish a link between individual bacterial cells from groundwater and the metabolism of a specific carbon source (naphthalene). The combination of these three techniques provided a clear advantage: SIP enriched the biomass of active microorganisms with ${ }^{13} \mathrm{C}$-stable isotope, Raman microscopy quantified the amount of stable-isotope carbon incorporated into cellular components of individual microbial cells (for example, DNA, RNA and amino acids) and FISH confirmed the phylogenetic affiliations of the very same cells.

Raman microscopy examines the scattering of laser light by the chemical bonds of different cell biomarkers. The proportion of stable isotope incorporation by cells affects this light scatter, resulting in measurable peak shifts for labelled cellular components. Raman-FISH does not require the use of radioisotopes, operates at the maximum resolution $(\sim 1 \mu \mathrm{m})$ of microautoradiography and FISH (FISH-MAR; also known as STAR-FISH (substrate- tracking autoradiography); Lee et al., 1999; Ouverney and Fuhrman, 1999), tracks the incorporation of ${ }^{13} \mathrm{C}-$ label into multiple cell biomarkers, and enables SIP incubations and subsequent microscopic analysis of cells from the same samples.

In the first demonstration of Raman-FISH, Huang et al. provide an elegant proof-of-concept with pure cultures and groundwater samples exposed to ${ }^{13} \mathrm{C}_{6}$ glucose and ${ }^{13} \mathrm{C}_{10}$-naphthalene, respectively. Their results demonstrated a quantitative measure of ${ }^{13} \mathrm{C}$ incorporation into the biomass of individual cells, identified those same cells with Pseudomonas spp. FISH probes and thus confirmed results obtained with enrichment and isolation work from the same groundwater environment.

The advent of techniques such as Raman-FISH is timely. Increasingly, research in microbial ecology is pushing back long-held paradigms and expanding the questions being asked of natural microbial communities. For example, as we begin to explore the role of newly described Archaea in the biogeochemical cycling of carbon and nitrogen, attempt to understand the microbiome of the human body, and seek to determine the function of communities of microbes inhabiting unlikely and seemingly uninhabitable niches, tools such as Raman-FISH have the potential to track the flow of carbon and nitrogen into individual cells, without the requisite of cultivation.

We anticipate that the potential of Raman-FISH will be best realized once several limitations are circumvented and some remaining questions are answered. For example, how does the sensitivity of Raman-FISH compare to FISH-MAR? FISH-MAR involves the exposure of an environmental sample to radiolabelled $\left({ }^{14} \mathrm{C}\right.$-labelled $)$ growth substrate, followed by microscopic detection of individual cells by microautoradiography and FISH. A key strength of FISH-MAR is its extreme sensitivity, enabling initial DNA- or RNA-SIP results to be confirmed in an almost natural incubation with in situ concentrations of substrate. The recent RamanFISH work described by Huang et al. (2007) monitored the incorporation of stable isotope label into phenylalanine, a central metabolite and essential amino acid. Cell phenylalanine probably labels more quickly than nucleic acids, possibly on the 
order of seconds or minutes (AS Whiteley, personal communication). Ideally, further validation could include a direct comparison of the sensitivities of Raman-FISH and FISH-MAR. Ultimately, the user may need to choose between alternative analysis platforms: the ultimate sensitivity of FISH-MAR or the convenience of combining SIP and Raman-FISH on the same experimental samples or better still, using both approaches in tandem (Raman-FISH ${ }^{2}$ MAR).

Other limitations common to all FISH-based analyses include (a) soil and sediment microbial communities are difficult to process using FISH and (b) hybridized cells must be relatively abundant for microscopic detection (for example, >1 in 1000 cells). As such, initial Raman-FISH analyses might focus on low diversity aquatic-based environments. However, recent work has demonstrated the retrieval and sorting of cells from soil followed by FISH hybridization (Podar et al., 2007), suggesting that Raman-FISH may be extended to virtually any environment of interest. As for the relative abundance limitation, without prior enrichment, particular microorganisms involved in the use of particular environmental substrates (for example, chemoautotrophic bacteria and Archaea) may be missed altogether. Coupling FISH, fluorescenceactivated cell sorting (FACS; Wallner et al., 1997; Podar et al., 2007) and Raman microscopy could make this approach universally applicable but would increase the technical difficulty of performing such an experiment.

Raman-FISH is relatively expensive. Currently, the required equipment includes a confocal Raman microscope with an integrated epifluorescence microscope. This 'basic' system can be assembled for approximately £230K, thus potentially not beyond the reach of larger well-equipped research facilities in environmental microbiology.

van Leeuwenhoek would certainly be pleased with the usefulness of Raman-FISH and its potential contribution to microbial ecology. The equipment's cost might have him turning over in his grave, but writing a grant application to obtain one nonetheless.
JD Neufeld is at Department of Biology, University of Waterloo, Waterloo, Ontario, Canada, E-mail: jneufeld@uwaterloo.ca and JC Murrell is at Department of Biological Sciences, University of Warwick, Coventry, UK. E-mail: j.c.murrell@warwick.ac.uk

\section{References}

Huang WE, Griffiths RI, Thompson IP, Bailey MJ, Whiteley AS. (2004). Raman microscopic analysis of single microbial cells. Anal Chem 76: 4452-4458.

Huang WE, Stoecker K, Griffiths R, Newbold L, Daims H, Whiteley AS et al. (2007). Raman-FISH: combining stable-isotope Raman spectroscopy and fluorescence in situ hybridization for single cell analysis of identity and function. Environ Microbiol 9: 1878-1889.

Lee N, Nielsen PH, Andreasen KH, Juretschko S, Nielsen JL, Schleifer KH et al. (1999). Combination of fluorescent in situ hybridization and microautoradiography - a new tool for structure-function analyses in microbial ecology. Appl Environ Microbiol 65: 12891297.

Neufeld JD, Wagner M, Murrell JC. (2007). Who eats what, where and when? Isotope labelling experiments are coming of age. ISME J 1: 103-110.

Ouverney CC, Fuhrman JA. (1999). Combined microautoradiography - 16S rRNA probe technique for determination of radioisotope uptake by specific microbial cell types in situ. Appl Environ Microbiol 65: 17461752.

Podar M, Abulencia CB, Walcher M, Hutchison D, Zengler $\mathrm{K}$, Garcia JA et al. (2007). Targeted access to the genomes of low-abundance organisms in complex microbial communities. Appl Environ Microbiol 73: 3205-3214.

Radajewski S, Ineson P, Parekh NR, Murrell JC. (2000). Stable isotope probing as a tool in microbial ecology. Nature 403: 646-649.

Wagner M, Horn M, Daims H. (2003). Fluorescence in situ hybridisation for the identification and characterization of prokaryotes. Curr Opin Microbiol 6: 302-309.

Wallner G, Fuchs B, Spring S, Beisker W, Amann R. (1997). Flow sorting of microorganisms for molecular analysis. Appl Environ Microbiol 63: 4223-4231. 